\title{
Discovery and Full Genome Characterization of SARS-CoV-2 in Stool Specimen from a Recovered Patient, China
}

\author{
YONGDONG LI ${ }^{1}$, YI CHEN ${ }^{1}$, HONGXIA NI ${ }^{1}$, BO YI ${ }^{1}$, DANDAN ZHANG ${ }^{1}$, JIANING ZHANG ${ }^{2}$, \\ WENJING WANG ${ }^{2}$, YUHUI LIU ${ }^{1}$, SULI JIAO ${ }^{1}$, GUOZHANG XU ${ }^{1 \star}$ and WEIDONG QIAN ${ }^{2 *}$ \\ ${ }^{1}$ Ningbo Municipal Center for Disease Control and Prevention, Ningbo, P.R. China \\ ${ }^{2}$ School of Food and Biological Engineering, Shaanxi University of Science and Technology, Xian, P.R. China
}

Submitted 20 April 2021, revised 6 July 2021, accepted 8 July 2021

\begin{abstract}
SARS-CoV-2 was found in a recovered patient's stool specimen by combining quantitative reverse transcription PCR (qRT-PCR) and genome sequencing. The patient was virus positive in stool specimens for at least an additional 15 days after he was recovered, whereas respiratory tract specimens were negative. The discovery of the complete genome of SARS-CoV-2 in the stool sample of the recovered patient demonstrates a cautionary warning that the potential mode of the virus transmission cannot be excluded through the fecal-oral route after viral clearance in the respiratory tract.
\end{abstract}

Keyword s: COVID-19, SARS-CoV-2, genome sequencing, recovered patient

The recent emergence of a novel coronavirus (SARSCoV-2) in China was associated with severe human infection and has emerged globally as a highly virulent human pathogen, causing severe public health concerns (Holshue et al. 2020; Zhu et al. 2020). Currently, the virus transmission is considered the following two main routes: respiratory droplets and contact (To et al. 2020). To fortify the response against the alarming levels of spread of the virus, many countries have tightened the detection of people showing symptoms and close contacts and have introduced necessary and urgent actions to prevent its spread (Wilder-Smith et al. 2020). The common symptoms of SARS-CoV-2 include fever, dry cough, shortness of breath or difficulty breathing, and myalgia (Chen et al. 2020). Several confirmed cases of COVID-19 with recurrently positive SARS-CoV-2 ribonucleic acid (RNA) in swabs and stool have been identified in China (Wu et al. 2020). We reported the discovery and complete genome characterization of SARSCoV-2 in the stool sample from a recovered patient.

A 26-year-old man lived in Wuhan, China, and denied exposure to the Huanan seafood market or wild animals. As shown in Fig. 1, the man got a headache without other evident symptoms, including fever or cough, on January 17, 2020. The next day, he developed a dry cough, nasal congestion, fatigue, muscle ache, and sore throat symptoms, with a body temperature of $37.6^{\circ} \mathrm{C}$. About $36 \mathrm{~h}$ after the onset of symptoms, he did not go to the local hospital for diagnosis and received no treatment at all. On January 20, 2020, he came to the fever clinic of the People's Hospital of Yuyao City after he took 6 hours from Wuhan to Ningbo, Zhejiang province, by high-speed rail. Given his travel history, he was admitted immediately to an airborne isolation unit as a suspected case of SARS-CoV-2.

On admission, high-resolution computed tomography of the chest was performed immediately, images of the patient demonstrated scattered cloud-wool inflammatory changes in the upper and lower lobes of the right lung and the lower lobe of the left lung. Meanwhile, the physical examination revealed that the patient was presented signs of infection; he was tachycardic, and his body temperature was $38.5^{\circ} \mathrm{C}$, while the patient was breathing ambient air. Results of the blood routine

\footnotetext{
* Corresponding authors: G. Xu, Ningbo Municipal Center for Disease Control and Prevention, Ningbo, P.R. China; e-mail:xugz@nbcdc.org.cn

W. Qian, School of Food and Biological Engineering, Shaanxi University of Science and Technology, Xỉan, P.R. China; e-mail: qianweidong@sust.edu.cn (c) $2021 \mathrm{Y}$. Li et al.
}

This work is licensed under the Creative Commons Attribution-NonCommercial-NoDerivatives 4.0 License (https://creativecommons. org/licenses/by-nc-nd/4.0/). 


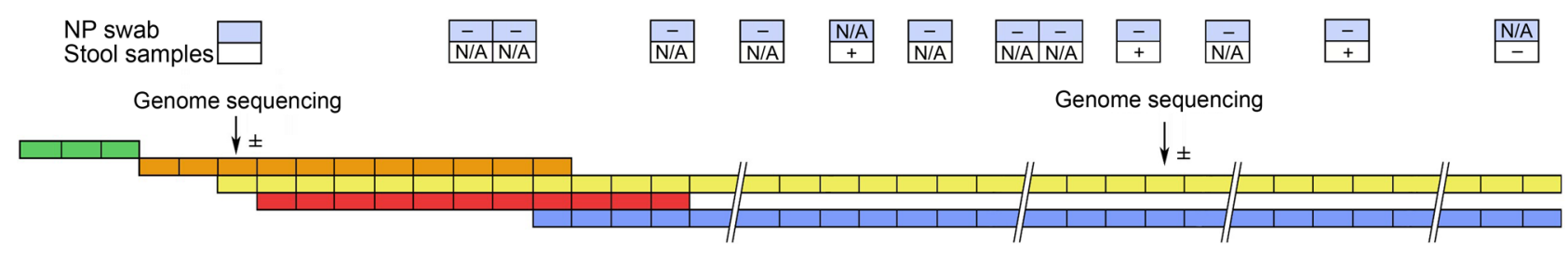

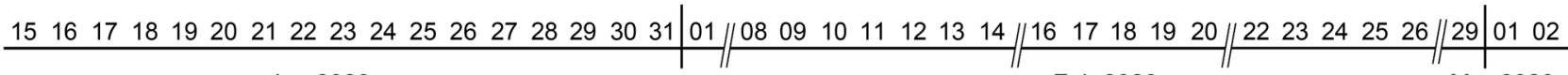
Jan 2020

Feb 2020

Mar 2020

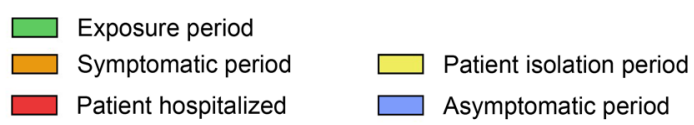

Fig. 1. Periods of COVID-19 in the patient with diagnosed coronavirus disease 2019.

showed a leukocyte count of $6 \times 10^{9} / 1$, a neutrophils level of $65.8 \%$, a lymphocyte percentage of $20.6 \%$, and a hypersensitive-C-reactive protein level of $0.6 \mu \mathrm{g} / \mathrm{ml}$.

For the quantitative RT-PCR (qRT-PCR) test, RNA was extracted from the samples using Magnetic Beads RNA Extraction Kit (Health Gene Technologies, Ningbo, China) according to the manufacturer's protocol. The samples with a Ct scores less than 40 were considered positive. In this study, two negative controls, and one positive control were randomly placed in samples to detect at the same time. On 21 January, he was confirmed positive for SARS-CoV-2 by qRTPCR after two sequential positive respiratory tract sample results, while the nasopharyngeal swab and stool specimens were negative. The results of the qRT-PCR test for influenza A and B, parainfluenza, respiratory syncytial virus, adenovirus, Mycoplasma pneumoniae, enterovirus, human metapneumovirus and bocavirus, Chlamydia pneumoniae, and Rickettsia burneti were negative. According to the diagnostic criteria in China (General Office of National Health Commission 2020), he was confirmed as a COVID-19 patient. The patient's symptoms of diseases caused by SARS-CoV-2 presented with progression to typical signs on day 9 of symptomatic treatment and antimicrobial therapy. During treatment, oropharyngeal swab samples of SARSCoV-2 RNA were performed repeatedly for surveillance and returned with negative results on 26 January. On 31 January, the patient met hospital discharge criteria or discontinuation of quarantine and was requested to extend the quarantine beyond 14 days at home (Lan et al. 2020). However, on February 11, the stool specimen obtained on negative day 17 later tested positive for SARS-CoV-2 by qRT-PCR (the viral load of $3.2 \log 10$ RNA copies/ml, ORF1ab Ct 30.8; nucleoprotein gene Ct 31.7), including persistent high levels (Ct values, 23 to 24), whereas the serum, nasopharyngeal, and sputum specimens remained negative. The stool sample obtained on positive-recurrent days 10 showed a trend toward decreasing levels of virus. On 25 February, the stool specimen tested negative for SARS-CoV-2 on positive-recurrent day 14. In the hospital, a routine blood test performed on February 13 revealed that cell counts fell within reference ranges, and a computed tomography scan on February 15 demonstrated that there were no identifiable abnormalities. During February 11-25, the man had no signs or symptoms and no detectable fever as well as other clinical signs or symptoms consistent with SARS-CoV-2 despite the positive results for SARS-CoV-2 in the stool samples, while nasopharyngeal and sputum specimens were negative.

Total nucleic acids were extracted from qRT-PCRpositive samples (SARS-CoV-2-016, the oropharyngeal samples obtained on 20 January and SARS-CoV-2-4794, the stool samples obtained on February 19) using the QIAamp Viral RNA Mini Kit (Qiagen, Germany) and then employed for whole-genome next-generation sequencing using the Illumina MiSeq platform (Illumina, USA). Double-stranded DNA for sequencing library preparation was performed by random priming using the Maxima H Minus Double Stranded cDNA Synthesis Kit (Thermo Fisher Scientific, Waltham, USA) with $2.5 \mu \mathrm{M}$ random hexamers according to the manufacturer's protocol. Sequence assembly was completed with the use of CLC Genomics Workbench version 20.0.2 (Qiagen, Germany). For the examination of the mutation profile within the SARS-CoV-2 genome and phylogenetic relationship, complete genome sequences of SARS-CoV-2-016 and SARS-CoV-2-4794 were aligned against publicly available SARS-CoV-2 sequences from NCBI using Minimap2 aligner (Li 2018). The results show that the full genome sequences from oropharyngeal and stool specimens were identical to one another and were nearly identical to other available SARS-CoV-2 sequences. The consensus sequence of SARS-CoV-2 was the same for two samples, suggesting that the virus can spread beyond the respiratory system to lurk in feces. In addition, there were only seven nucleotides and two amino acid that differed at nps 3 of orflab and ORF8 between this patient's virus and the 
SARS-CoV-2 reference sequence (NC_045512.2). Phylogenetic analysis revealed that the two SARS-CoV-2 genomes in this study are in the A clade. The genome sequences are accessible through GenBank (accession numbers MT318828 and MT318829).

Although respiratory transmission is considered the primary route for SARS-CoV-2 and the evidence is yet insufficient to establish practical measures for the group of patients recovered from COVID-19, who tested negative for respiratory tract sample results but positive for fecal samples. Wu et al. (2020) reported that a few patients' fecal samples remained positive for SARS-CoV-2 RNA for a mean of 11.2 days after respiratory tract samples became negative by qRT-PCR. Similarly, the direct evidence showed that SARS-CoV-2 was identified in 44 stool specimens (44 out of 153) (Wang et al. 2020). Here, we reported that a recovered patient tested positive for SARS-CoV-2 in stool specimens for at least an additional 15 days after he was recovered, whereas respiratory tract specimens were negative. These phenomena observed might be attributed to the fact that ACE2, the entry receptor for SARS-CoV-2, is highly expressed in the glandular cells of gastric, duodenal, and rectal epithelia (Hoffmann et al. 2020).

Determining whether SARS-CoV-2 exists in a complete or fragmented manner by qRT-PCR is difficult. In addition, determining whether mutations of the virus that can adapt to the human host occur as long as the virus continues to circulate in humans by qRT-PCR is difficult. Here, genome sequencing was applied to profile the genome sequence of the virus in the samples from different dates of symptom onset. The full genome sequences from two samples before and after recovery were identical to one another, indicating that direct evidence was identified genetically supporting the existence of the move of the virus from the respiratory tract into the gastrointestinal tract in the recovered patient, which may discharge the stool with the virus for a long time. Additionally, our study also suggests that the virus might become more adapted in the human body as a human disease. The genome data of the virus in the stool sample may contribute to further investigations of the evolution of the virus (Shen etal. 2020). However, the quantitative relationship between viral relative abundance/titer and infection is unclear.

Overall, our study has revealed and identified the full genome of SARS-CoV-2 in the stool sample of a recovered patient, an uncommon feature possessed by an RNA virus. Whether the virus, including the complete genome hidden in the body, can replicate and thereby cause the transmission via the fecal-oral route, awaits further investigation. However, a possible therapy for this could be extended use of antiviral agents or in combination with traditional Chinese medicine
(TCM) for recovered patients for further two weeks. Compared with Western medicine, treating patients as an organic whole is the prerequisite for TCM to treat all diseases (Zhao et al. 2021). In addition to the COVID19 vaccines currently in use, in the absence of specific drugs globally, China's experience in the application of TCM can be used as a valuable reference to treating COVID-19 (Zhao et al. 2021). Meanwhile, there is a potential need to establish the guideline for surveillance and adequate disinfection in latrines from the recovered patient.

\section{Ethical statement}

This study was approved by Ningbo municipal center for disease control and prevention ethical review committee (202001).

\section{Author contributions}

Conceptualization: Guozhang Xu, Weidong Qian; Methodology: Yongdong Li, Guozhang Xu; Formal analysis and investigation: Yi Chen; Writing - original draft preparation: Weidong Qian, Hongxia Ni; Writing - review and editing: Dandan Zhang, Jianing Zhang, Wenjing Wang; Funding acquisition: Bo Yi; Resources: Yuhui Liu, Yongdong Li; Supervision: Suli Jiao, Weidong Qian. All authors contributed to manuscript revision and approved the final version.

\section{Funding}

This study was funded by the Science and Technology Research Project of Ningbo Science and Technology Bureau (No: 2020C50001).

\section{Conflict of interest}

The authors do not report any financial or personal connections with other persons or organizations, which might negatively affect the contents of this publication and/or claim authorship rights to this publication.

\section{Literature}

Chen N, Zhou M, Dong X, Qu J, Gong F, Han Y, Zhang L. Epidemiological and clinical characteristics of 99 cases of 2019 novel coronavirus pneumonia in Wuhan, China: a descriptive study. Lancet. 2020;395(10223):507-513.

https://doi.org/10.1016/S0140-6736(20)30211-7

Hoffmann M, Kleine-Weber H, Schroeder S, Kruger N, Herrler T, Erichsen S, Pohlmann S. SARS-CoV-2 cell entry depends on ACE2 and TMPRSS2 and is blocked by a clinically proven protease inhibitor. Cell. 2020;181(2):271-280.

https://doi.org/10.1016/j.cell.2020.02.052

Holshue ML, DeBolt C, Lindquist S, Lofy KH, Wiesman J, Bruce H. First case of 2019 novel coronavirus in the United States. N Engl J Med. 2020;382(10):929-936.

https://doi.org/10.1056/NEJMoa2001191

Lan L, Xu D, Ye G, Xia C, Wang S, Li Y, Xu H. Positive RT-PCR test results in patients recovered from COVID-19. JAMA. 2020; 323(15):1502-1503. https://doi.org/10.1001/jama.2020.2783

Li H. Minimap2: pairwise alignment for nucleotide sequences. Bioinformatics. 2018; 34(18):3094-3100.

https://doi.org/10.1093/bioinformatics/bty191

Shen Z, Xiao Y, Kang L, Ma W, Shi L, Zhang L, Li M. Genomic diversity of SARS-CoV-2 in coronavirus disease 2019 patients. Clin Infect Dis. 2020;71(15):713-720. https://doi.org/10.1093/cid/ciaa203 
To KK, Tsang OT, Chik-Yan Yip C, Chan KH, Wu TC, Chan JMC, Yuen KY. Consistent detection of 2019 novel coronavirus in saliva. Clin Infect Dis. 2020;71(15):841-843.

https://doi.org/10.1093/cid/ciaa149

Wang W, Xu Y, Gao R, Lu R, Han K, Wu G, Tan W. Detection of SARS-CoV-2 in different types of clinical specimens. JAMA. 2020 323(18):1843-1844.

https://doi.org/10.1001/jama.2020.3786

Wilder-Smith A, Chiew CJ, Lee VJ. Can we contain the COVID-19 outbreak with the same measures as for SARS? Lancet Infect Dis 2020;20(5):102-107.

https://doi.org/10.1016/s1473-3099(20)30129-8
Wu Y, Guo C, Tang L, Hong Z, Zhou J, Dong X, Huang X. Prolonged presence of SARS-CoV-2 viral RNA in faecal samples. Lancet Gastroenterol Hepatol. 2020;5(5):434-435. https://doi.org/10.1016/S2468-1253(20)30083-2

Zhao Z, Li Y, Zhou L, Zhou X, Xie B, Zhang W, Sun J. Prevention and treatment of COVID-19 using traditional Chinese medicine: A review. Phytomedicine. 2021;85:153308. https://doi.org/10.1016/j.phymed.2020.153308

Zhu N, Zhang D, Wang W, Li X, Yang B, Song J, Research T. A novel coronavirus from patients with pneumonia in China, 2019. N Engl J Med. 2020;382(8):727-733.

https://doi.org/10.1056/NEJMoa2001017 